\title{
A Survey of the Role of Community Pharmacists in Dhaka city, Bangladesh
}

\author{
Abdur Rakib, Md. Shahid Sarwar, Shaheda Zannah, Sayema Khanum and Mamunur Rashid \\ Department of Pharmacy, Southeast University, Banani, Dhaka-1213, Bangladesh
}

Received: May 10, 2015; Accepted: June 15, 2015; Published (Web): July 21, 2015

\begin{abstract}
Community pharmacists play a vital role in improving medication safety and better healthcare facility to the community. Unfortunately, the field of community pharmacy in Bangladesh is still to be developed and there is scarcity of data regarding current role of community pharmacists in Bangladesh. The present study was conducted to find out the role of community pharmacists in Bangladesh. For the study purpose, we visited 42 retail pharmacies in Banani, Khilkhet and Uttra areas of Dhaka city and interviewed the community pharmacists working there. We found that there were total 50 community pharmacists in 42 retail pharmacy and also observed that most of the pharmacists (64\%) worked alone. Interestingly, we didn't find a single A-grade pharmacist working as community pharmacist. B-grade pharmacists who completed a 3 year diploma in pharmacy were only 4 in number and $92 \%$ of them were C-grade pharmacists with 3-4 months training. The average age of the participants was $29.6 \pm 1.2$ years. The average length of service for the community pharmacists was $7.6 \pm 1.3$ years. The top issues that patients consulted with the pharmacists were on medication use (76\%), which physician they should visit (54\%) and therapy (26\%). There was not a significant effect of work experience of community pharmacists on their attitudes toward community-based clinical services. In this study, we observed that $95 \%$ community pharmacies provide blood pressure measurement facility, $90 \%$ provide diabetes screening, $76 \%$ provide dressing services, and only $47 \%$ provide nebulization. No community pharmacist was found to be involved with adverse drug reaction reporting. In conclusion, we recommend that the government and the pharmacy regulatory authority should take sufficient initiatives to develop the community pharmacy sector in Bangladesh for the welfare of mankind.
\end{abstract}

Key words: Community pharmacists, retail pharmacy, patient care, Bangladesh

\section{Introduction}

Everyday millions of people across the world seek their health care needs to the community pharmacists. Patients rely on community pharmacists for counselling because they are the most available and trusted healthcare providers (Yegenoglu et al., 2005). Currently, pharmacists are shifting their role from a drug-focused approach towards a patient centred approach with the aim of achieving better outcomes from drug therapy (Poudel et al., 2009). In addition, due to the continuous progress in pharmacy profession, the scope of this profession is changing from drug manufacturing and quality management to providing drug information and patient care (Yegenoglu et al., 2005). But yet not all roles of community pharmacy services beyond medication advice or supply have been utilised to their full potential. Pharmacists and other dispensers are in many cases the final link between the medication and the patient. Community pharmacies have always been a major location for health care worldwide. Pharmacy employees are consulted for health advice on problems of all kinds, and remedies are sold or dispensed with almost every transaction. Some of the remedies are safe and effective when used correctly but otherwise can be dangerous; others are ineffective no matter how they are used (Matheson et al., 2002; Adepu and Nagavi, 2006).

Bangladesh, the seventh most populous country (with about 161 million people) in the world and it is estimated that the population of the country will become nearly double by 2050 (Streatfield and Karar, 2008). With such a huge population density $\left(1142.29 / \mathrm{Km}^{2}\right)$, there are only fewer numbers of pharmacists who have been serving in hospitals and community pharmacies. The insufficiency of number of qualified pharmacists forces the patient toward

Correspondence to: Mamunur Rashid; E-mail: mamun69jp@yahoo.com 
inappropriate use of medication either from the error in the prescription by the physician or from the self-medication. Irrational drug therapies may causes unproductive and perilous treatment, exacerbation or prolongation of illness, distress and harm to patient, increased burden of treatment. The most common causes of irrational use of drugs are lack of information, imperfect and insufficient training and education of medical graduates, poor communication between health professionals and patients, lack of diagnostic facilities or uncertainty of diagnosis, demand from patient, defective drug supply or ineffective drug regulation, and promotional activities of pharmaceutical industries (Shivhare et al., 2010). Considering all these factors, the present study was designed to identify the current status of community pharmacy services and role of community pharmacists in different selected areas of Dhaka city, Bangladesh.

\section{Methods}

In our study, we tried to evaluate the role of community pharmacists in the several retail pharmacies in Banani, Khilkhet and Uttara areas of Dhaka city. For this purpose, each retail pharmacist was interviewed with a structured questionnaire. The questionnaire was designed with the educational background, age, sex, experience and grade of the community pharmacists, their knowledge about the drugs, drugs storage and various services and advices they provide to the patients and customers. The community pharmacists who denied to participate and who disagreed to share the necessary information were excluded from the study.

\section{Results}

Study subjects: Total 42 retail pharmacies from three different regions of Dhaka city including Banani, Khilkhet and Uttara were included in this study. In most retail pharmacy (64\%) shops, pharmacists worked alone. 50 pharmacists working in these retail pharmacies were included in this study. As the sector of community pharmacy is not well developed in Bangladesh, we didn't find a single A-grade pharmacist (Table 1). B-grade pharmacists who completed a 3 years diploma in pharmacy were only 4 in number, whereas the remaining of them were C-grade pharmacists (92\%).
Table 1. Total number of study subjects.

\begin{tabular}{lc}
\hline Study subjects & Number \\
\hline Total number of pharmacy studied & 42 \\
Total number of working pharmacists & 50 \\
A grade pharmacists & 0 \\
B grade pharmacists & 4 \\
C grade pharmacists & 46 \\
\hline
\end{tabular}

Demographic characteristics of the community pharmacists: The average age of the participants was $29.6 \pm 1.2$ years. The average length of service for the community pharmacists was $7.6 \pm 1.3$ years (Figure 1). Among 42 retail pharmacists, only $16 \%$ pharmacists completed their graduation, whereas $62 \%$ and $22 \%$ completed HSC and SSC respectively (Figure 2). Demographic characteristics of respondents are presented in table 2.

Table 2. Demographic characteristics of the community pharmacists.

\begin{tabular}{lcc}
\hline & Characteristics & Respondents (n) \\
\hline Sex $(\mathrm{n}=50)$ & Female & 0 \\
& Male & 50 \\
\hline Experience (years) & $0-5$ & 28 \\
& $6-10$ & 12 \\
& $11-15$ & 8 \\
& $15+$ & 2 \\
\hline Education & SSC & 11 \\
& HSC & 31 \\
& Hons & 8 \\
\hline
\end{tabular}

Issues that the patients discussed with community pharmacists: Participating pharmacists were given an open-ended question to offer topics on which their patients in the community setting consult them. "Name of the drug, directions for usage, side effects and duration of use" were classified as "medication use", "information about their current treatment" as "therapy", and "which physician to see for their ailments" as "physician". The top issues that patients consulted the pharmacists were on medication uses $(76 \%)$, which physician they should visit $(54 \%)$ and therapy (26\%) (Table 3).

Awareness of community pharmacists about their job responsibilities: There was not a significant effect of work experience of community pharmacists on their attitudes toward community-based clinical services. Table 4 shows 
the awareness of community pharmacists toward their role in community based clinical services.

Key services of retail pharmacy: In this study, we observed that community pharmacists in the study area are not effective in managing and following the drug therapy for patients. Only $4 \%$ community pharmacists informed the patient about the duration of drug use, drug name and different features and side effects of drugs. Only $2 \%$ pharmacists informed about storage condition. Table 5 shows the facilities of retail pharmacy provided for the patients in Banani, Khilkhet and Uttra area.

Table 3. Issues that patients discussed with community pharmacists.

\begin{tabular}{lc}
\hline Issues discussed & $\mathrm{N}(\%)$ \\
\hline Medication used & $38(76)$ \\
Therapy & $13(26)$ \\
Physician & $27(54)$ \\
Health in general & $8(16)$ \\
Diagnosis & $2(4)$ \\
\hline
\end{tabular}

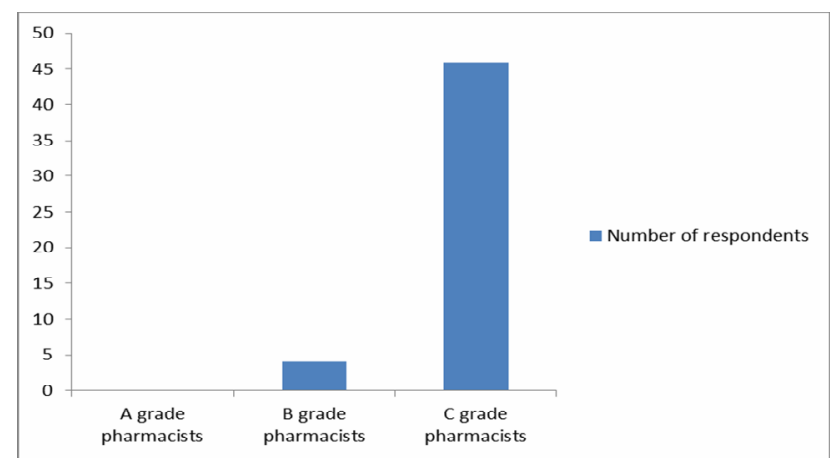

Figure 1. Different grades of pharmacist among the respondents.

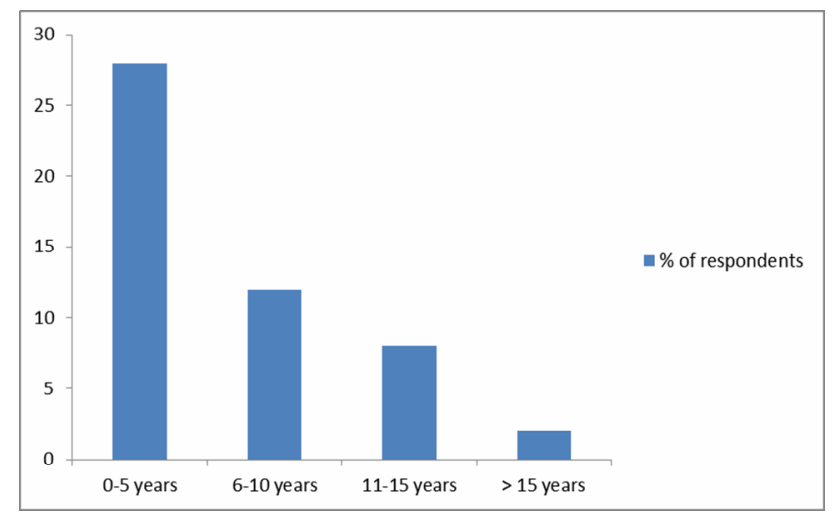

Figure 2. Experiences of community pharmacists.

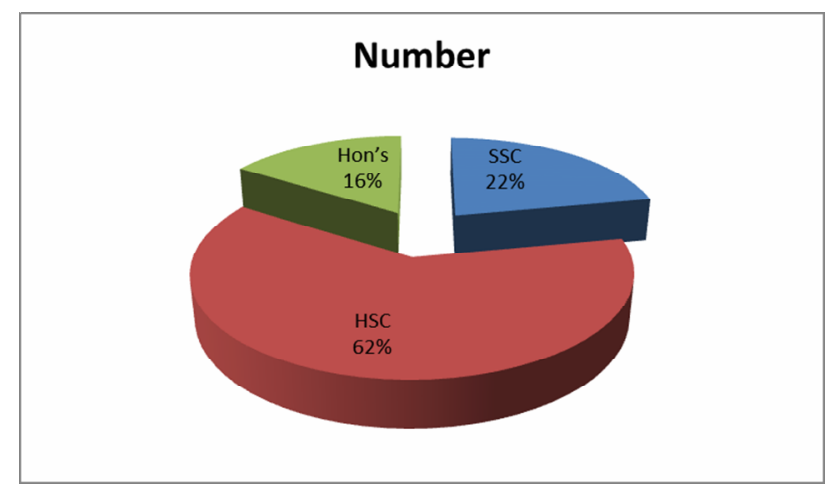

Figure 3. Educational qualification of the community pharmacists.

Table 4. Awareness of community pharmacists about their job responsibilities.

\begin{tabular}{lcc}
\hline Responsibilities & \multicolumn{2}{c}{ Responses (\%) } \\
\cline { 2 - 3 } & Yes & No \\
\hline Consulting and informing doctors and other health personnel & 8 & 92 \\
Taking drug history of the patient & 12 & 88 \\
Explaining the test results to the patient & 14 & 86 \\
Consultancy on OTC drugs (self therapy) & 22 & 78 \\
Counseling about drug interactions & 2 & 98 \\
Give antibiotics without prescription & 82 & 18 \\
Give sedative and narcotic drugs without prescription & 80 & 20 \\
Being informed about health risks & 12 & 88 \\
Counseling about social education themes & 2 & 98 \\
Reporting adverse drug reaction & 0 & 100 \\
\hline
\end{tabular}


Table 5. Key services provided by the community pharmacists.

\begin{tabular}{lcc}
\hline Subject analyzed & \multicolumn{2}{c}{ Responses $(\%)$} \\
\cline { 2 - 3 } & Yes & No \\
\hline Informing patients or relatives about the duration of drug use & 4 & 96 \\
Informing patients or relatives about drug administration route & 6 & 94 \\
Informing patients or relatives about drug dose and schedule & 2 & 98 \\
Informing patients or relatives about drug storage conditions & 2 & 98 \\
Informing patients or relatives about drug name and features & 4 & 96 \\
Warning patients or relatives about side effects of drugs & 4 & 96 \\
Informing patients on detrimental health effects of smoking & 8 & 92
\end{tabular}

Clinical screening by retail pharmacists: In this study, we observed that $95 \%$ retailer measure blood pressure, $90 \%$ provide diabetes screening, $76 \%$ provide dressing services and only $47 \%$ provide nebulization (Table 6).

Table 6. Clinical screening provided by the community pharmacists.

\begin{tabular}{lccc}
\hline \multirow{2}{*}{ Screenings } & \multicolumn{2}{c}{ Responses (n) } & \multirow{2}{*}{ Percentages } \\
\cline { 2 - 3 } & Yes & No & \\
\hline Blood pressure & 40 & 2 & $95 \%$ \\
Diabetes & 38 & 4 & $90 \%$ \\
Dressings & 32 & 10 & $76 \%$ \\
Nebulization & 20 & 22 & $47 \%$ \\
\hline
\end{tabular}

\section{Discussion}

Poor adherence to medication is a major problem among patients with chronic diseases (Martin et al., 2005). The pharmacist could be a coordinator between different members of healthcare team and the patients. Thus, involvement of pharmacists in health management system is becoming very crucial day by day. Community pharmacists come in direct contact with the public and they not only dispense medications but also counsels patients regarding general health topics such as diet, exercise, stress management, over-the-counter medications etc. Some community pharmacists also provide specialized services to help patients with diabetes, asthma, smoking cessation, drug addiction, and patients with high blood pressure (Shill and Das, 2011; Lipton et al., 1995).

In our study, we found that there are not sufficient number qualified community pharmacists working in our study area. However, we found that some people who completed their graduation involved themselves as community pharmacists. Although this was very small in number but this provide us new hope that A-grade pharmacists who completed their graduation in pharmacy might involve with this profession in near future. One of the main responsibilities of community pharmacists is to consult with physician and nurses for providing better healthcare facility to the patients (Albanese et al., 2010). Unfortunately, in our study we observed that limited number of pharmacists maintained communication with physician and other health professionals. Reporting medication histories are very essential to prevent prescription errors and subsequent risks to the patients. Accurate medication histories are also important in detecting drug-related pathology or changes in clinical signs that may be the result of drug therapy. A good medication history should encompass all currently and recently prescribed drugs, previous adverse drug reactions including hypersensitivity reactions, any over-the counter medications, including herbal or alternative medicines, and adherence to therapy (Gerald, 2009). This study found that community pharmacists were not interested either to document the previous drug history of the patients or to explain the results of their test reports.

Patients counselling about different drug-drug and drug-food interaction have paramount importance to avoid the possible undesirable effects (Hussain, 2011). But we found that only negligible numbers of pharmacists offer this service to the patients which are one of the major causes of the unwanted side effects and inferior drug action to the patients. We also observed that the community pharmacists have tendency to dispense antibiotic and sedative-hypnotic drugs without 
prescription. This type of tendency may lead to some potentially detrimental effect including antibiotic resistance, psychological and physiological dependency. The pharmacists were not also found to counsel the patients about duration of drug therapy, drug doses, route of administration, drug storage conditions, possible side effects, and detrimental effects of smoking. But the positive observation was that noticeable number of community pharmacies offer blood pressure measurement, diabetes screening, dressing service and nebulization facility to the patients which help the patients for their primary diagnosis and treatment processes.

However, this was a preliminary study conducted in few selected areas of Dhaka city which may not represent the actual scenario. We recommend further study with larger sample size in different region of Bangladesh to find out current role of community pharmacist in Bangladesh.

\section{Conclusion}

In our study, we found that the community pharmacy profession in Bangladesh is still at the very primary stage. The government and pharmacy regulatory authority should take sufficient initiative to develop the community pharmacy sector in Bangladesh. There is a gap in the knowledge of community pharmacists regarding medicine safety, usage and storage, which is having profound impact on the outcomes of therapy. This is partly due to lake of properly educated and trained pharmacists. Therefore, there is an urgent need to promote the community pharmacy profession for helping the patients to use medicines in safe and effective manner.

\section{References}

Adepu, R. and Nagavi, B.G. 2006. General practitioner's perceptions about the extended roles of the community pharmacist in the state of Karnataka: A study. Indian J. Pharm. Sci. 68, 36-40.
Albanese, N.P., Rouse, M.J. and Council on Credentialing in Pharmacy. 2010. Scope of contemporary pharmacy practice: roles, responsibilities, and functions of pharmacists and pharmacy technicians. J. Am. Pharm. Assoc. 50, e35-69.

Gerald, R.J.F. 2009. Medication errors: the importance of an accurate drug history. Br. J. Clin. Pharmacol. 67, 671-675.

Hussain, M.S. 2011. Patient counseling about herbal-drug interactions. Afr. J. Tradit. Complement. Altern. Med. 8, 152-163.

Lipton, H.L., Byrns, P.J., Soumerai, S.B. and Chrischilles, E.A. 1995. Pharmacists as agents of change for rational drug therapy. Int. J. Technol. Assess. Health. Care 11, 485-508.

Martin, L.R., Williams, S.L., Haskard, K.B. and DiMatteo, M.R. 2005. The challenge of patient adherence. Ther. Clin. Risk Manag. 1, 189-199.

Matheson, C., Bond, C.M. and Pitcairn, J. 2002. Community pharmacy services for drug misusers in Scotland: what difference does 5 years make? Addiction. 97, 1405-1411.

Poudel, A., Khanal, S., Alam, K. and Palaian, S. 2009 Perception of Nepalese community pharmacists towards patient counseling and continuing pharmacy education program: a multicentric study. J. Clin. Diagn. Res. 3, 14081413.

Shill, M.C., and Das A.K. 2011. Medication practices in Bangladesh- roles of pharmacists at current circumstances. Int. J. Pharm. Pharm. Sci. 3, 5-8.

Shivhare, S.C., Kunjwani, H.K., Manikrao, A.M. and Bondre, A.V. 2010. Drugs hazards and rational use of drugs: a review. J. Chem. Pharm. Res. 2, 106-112.

Streatfield, P.K. and Karar, Z.A. 2008. Population challenges for Bangladesh in the coming decades. J. Health Popul. Nutr. 26, 261-272.

Yegenoglu, S. and Ozcelikay, G. 2005. Counseling of pharmacists to community on issues other than drug purchasing and drug related information: a survey in Ankara. Turkish. J. Pharm. Sci. 2, 83-91. 